\title{
EXTREME EIGENVECTORS OF A NORMAL OPERATOR
}

\section{S. J. BERNAU}

In a recent paper [3] MacCluer proves that if $A$ is a bounded normal operator on a complex Hilbert space $H$ then the extreme points of the numerical range of $A$ are eigenvalues of $A$. MacCluer's proof uses the spectral theorem in an essential fashion. Our purpose is to point out that a slightly stronger result is elementary, independent of the spectral theorem and true for unbounded normal operators. We show that if $\|x\|=1$ and $\lambda=(A x, x)$ is an extreme point of the numerical range then $A x=\lambda x$.

Theorem. Let $A$ be a not necessarily bounded, normal operator on a complex Hilbert space $H$, and let $W(A)=\{(A x, x): x \in D(A),\|x\|=1\}$. If $\lambda$ is an extreme point of $W(A), x \in D(A),\|x\|=1$ and $(A x, x)=\lambda$, then $A x=\lambda x$.

(Recall that a closed operator $A$ is normal if its domain $D(A)$ is dense in $H$ and $A A^{*}=A^{*} A$.)

Proof. Because $W\left(e^{i \theta}(A-\lambda I)\right)=e^{i \theta}(W(A)-\lambda)$ we may assume that $\lambda=0$ and $W(A)$ is contained in the closed right half plane $\{z: \operatorname{Re} z \geqq 0\}$. Clearly $A+A^{*}$ is symmetric and $W\left(A+A^{*}\right)$ is a set of nonnegative real numbers; thus, by the generalized Schwarz inequality $[4, \S 104]$.

(1) $\left|\left(\left(A+A^{*}\right) x, y\right)\right|^{2} \leqq\left(\left(A+A^{*}\right) x, x\right)\left(\left(A+A^{*}\right) y, y\right)(x, y \in D(A))$.

Suppose that $x \in \mathscr{D}(A),\|x\|=1$ and $(A x, x)=0$. It follows that $\left(A^{*} x, x\right)=0$ and hence, by $(1),\left(\left(A+A^{*}\right) x, y\right)=0(y \in D(A))$. Because $D(A)$ is dense, $\left(A+A^{*}\right) x=0$. Let $M$ be the (nontrivial) null space of $A+A^{*}$. For $y$ in $M$

$$
(A y, y)=\left(y, A^{*} y\right)=-(y, A y)=-(A y, y)^{*},
$$

so that $\operatorname{Re}(A y, y)=0(y \in M)$. Because 0 is an extreme point of $W(A)$ it follows that $\operatorname{Im}(A y, y)$ is either nonnegative, or nonpositive for all $y$ in $M$. Suppose that $\operatorname{Im}(A y, y) \geqq 0(y \in M)$. Then, for $S=-i A$ we have $(S y, y) \geqq 0(y \in M)$. Because $x \in D(A),\|x\|=1$ and $(A x, x)=0$ we have, $(S x, x)=0$ and, by the generalized Schwarz inequality again,

$$
(S x, y)=0 \quad(y \in M) .
$$

Received by the editors May 7, 1966. 
If $A$ is bounded we see at once that $M$ is closed and invariant under $A$. Hence $S x=-i A x \in M$ and $A x=0$.

If $A$ is not bounded we proceed as follows. Because $A$ is normal $\left(A+A^{*}\right)^{* *}$, the closure of $A+A^{*}$, is self adjoint. It follows that

$$
M^{\perp}=\operatorname{cl} \Re\left(\left(A+A^{*}\right)^{* *}\right)=\operatorname{cl} \Re\left(A+A^{*}\right) .
$$

Now let $z \in \mathscr{D}(A)$, by [2, Lemma XII.7.1], there is a sequence $\left(z_{n}\right)$ in $\mathfrak{D}\left(A^{*} A\right)$ such that $z_{n} \rightarrow z$ and $A z_{n} \rightarrow A z$. Because $A$ is normal $A^{*} z_{n} \rightarrow A^{*} z$ and hence

$$
\begin{aligned}
\left(S x,\left(A+A^{*}\right) z\right) & =\lim \left(-i A x,\left(A+A^{*}\right) z_{n}\right) \\
& =\lim \left(-i x, A\left(A+A^{*}\right) z_{n}\right) \\
& =\lim \left(-i x,\left(A+A^{*}\right) A z_{n}\right) \\
& =\lim \left(-i\left(A+A^{*}\right) x, A z_{n}\right) \\
& =0 .
\end{aligned}
$$

Thus $S x \in M^{\perp \perp}=\bar{M}$ and, by (2), $A x=i S x=0$. This proves our theorem.

In conclusion we remark that the result that, for normal $A$, $\left(A+A^{*}\right)^{* *}$ is self adjoint is given as an exercise in [2, XII.9.11]. An elementary proof can be based on the polar decomposition for a normal operator [2, XII.9.10]. This in turn depends on the existence of a square root for an unbounded positive self adjoint operator. An elementary proof of this and of the polar decomposition are given in $[1]$.

ADded IN Proof. The bounded case of our theorem is obtained, with essentially the same proof, by S. Hildebrandt, Über den numerischen Wertebereich eines Operators, Math. Ann. 163 (1966), 230-247 (Lemma 11) and is originally due to W. F. Donoghue, On the numerical range of a bounded operator, Michigan Math. J. 4 (1957), 261-263. Neither of these references was known to the author when this paper was written.

\section{REFERENCES}

1. S. J. Bernau, The square root of a positive self adjoint operator, J. Austral. Math. Soc. (to appear).

2. N. Dunford and J. T. Schwartz, Linear operators, Part II, Interscience, New York, 1963.

3. C. R. MacCluer, On extreme points of the numerical range of normal operators, Proc. Amer. Math. Soc. 16(1965), 1183-1184.

4. F. Riesz and B. Sz. Nagy, Functional analysis Ungar, New York, 1955.

University of Canterbury, Christchurch, New Zealand 